$$
\text { Francè Bezlaj }
$$

\title{
CONTRIBUTIONS LEXICOGRAPHIQUES
}

\section{SLOVENE DIALECTAL DOBRA _CONTREE OU L'EAU ABONDE,}

Dans les «Novice» du 6 avril 1859, p. 108, Fr. Pohorski (probablement un pseudonyme d'un auteur inconnu) publia des matériaux lexicographiques sous le titre «Quelques mots rares de Pohorje en Styrie». Cette contribution passa inaperçue et Pleteršnik ne cite dans son dictionnaire que quelques-uns de ces mots qu'il a tirés d'une autre source. Dans un passé plus récent, J. Kelemina prit dans ce recueil le nom commun tega, teha echalet de montagnes, die Taie en all. carinth. (Slovenski etnograf VI-VII 323) < *tegia, (pré)rom. attegia (Meyer-Lübke, REW 761) et sot «chemin de montagne» (SR VIII 88), ce qui pourrait venir, après avoir passé par le roman, de (pré)rom. "tsotto- fossé, excavations (J. Hubschmid, Pyrenäenmörter 36). Les autres mots cités par Pohorski n’ont pas encore été pris en évidence, bien qu'il y en ait plusieurs qui sont très intéressants; je me borne à citer mal, mel ssommet, colliss (cf. roum. mal «monts, alb. mal smonts, irl. anc. mell scollines, lette mala ebord, rives, parmi les toponymes p. ex. Malta en Carinthie, all. Maltein, au $\mathrm{X}^{\bullet}$ siècle Malontina), puis pen «cimes, kunj «sommet, kob, ceb «arrête de montagne, mons longe extensus», skarb, skrab, skrob slocus gibbosus, flexuosus (cf. le toponyme Skrabnik et le ruisseau Skrabska en Sty rie). Certains mots de ce recueil sont des expressions slaves oubliées, p. ex. namen, ramen «fort, puissant, attesté encore chez Megiser (Miklošič, Lex. Palaeoslav. 783) et dans l'adverbe rameno dans les chansons populaires (Štrekelj, SNP 14/9 et 453/92) < *orměno- (étymologie de Bruickner, KZ XLV 10z; Schnetz, JA XL 70 et Studi etruschi IV 217). Il n'y a aucune raison de douter de la véracité de l'auteur oublié, qui cependant n'a pas pu inventer des mots.

Le nom commun dobra contrée où l'ẹu abondes mérite l'attention particulière des slavisants. Pohorski ne mentionne pas le nom du lieu où il a trouvé ce mot, et ne le cite pas en transcription phonétique exacte; il nous est done impossible de retrouver la voyelle primitive de la racine

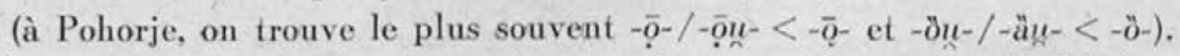
Du point de vue sémantique, on pourrait rapprocher ce mot de *dọbra, cf. v. tch. dúbra spiscina < dọbrava (Gebauer, SS I 352). L'idée d'un rapport entre cet appellatif slovène et le type de nom Dobrna, Dobrnica 
est toute proche; il est cependant difficile de faire dériver ces noms de dabrò «bons, puisque ces dérivés d'adjectifs ne se produicent jamais dans la toponymie slave.

Dans sa critique de la réimpression des études de toponymie de Miklošič, A. Brückner dit: «von den Namen, die unter dobró zusammenstehen, dürfte mancher zu dibrı gehören». Ceci vaut surtout pour le type dobrana qui concurrence dans une vaste région le type də̋brona. En Slovénie, nous avons plusieurs toponymes de ce type qui, depuis les temps les plus reculés, indiquent un -o-, comme p. ex. Dobrna près de Celje, 1155 Dobern, 1522 Doefer, 1387 Toefer (Zahn, ONB 135) et le ruisseau Dobrnica, ca. 1500 pach Dabernicza (Zahn, ibid.); on trouve un autre ruisseau Dobrnca près de Bela Cerkev en Basse-Carniole. Le toponyme Dobrnič près de Trebnje est noté en 1136 comme Dobernik, 1296 Doreer$n i k$, 1524 déjà Doevernik, 1328 Douernich (M. Kos, STL). Les noms qui ne sont pas cités dans les textes historiques exigent plus de circonspection; le top. Dobrna près de Trbovlje s'écrit en 1265 Dobringe (Zahn, $O N B$ 155) ce qui, comme Dobring, Dobryn en 1490 (Zahn, ONB 137), Debrin et Debrin-Bach dans la vallée supérieure de la Mura, 1243 Dobryn, 1250 Dobrin (Pirchegger, ON im Mürzgebiet 21), pourrait venir de l'anthroponyme *Dobrin-. On peut cependant supposer à côté du type *dobrona aussi *dobrina, ef. s.-cr. Dobrina à côté de Dàbrina (ARj II 217 et 516).

On trouve en slovène comme noms de lieux dits encore Dobrna et Dobrnič (recueil MSl ms.), et dans la vallée de la Möll en Carinthie on cite le nom de maison Dabernig (Kranzmayer, Carinthia 140, 1950, 607). Le vocalisme en - $e$ - n'est attesté que par le nom de l'alpe Debrnca en Carinthie, all. Dobernitz. Le nom de Dobrije, all. Döbriach en Carinthie fait lui aussi penser les germanistes à də̈brı (Kranzmayer, Carinthia, idem), bien que la formation suffixale de ce nom ne me semble pas claire (à la rigueur $<*$ dobrojane).

En serbo-croate, on trouve, dérivés du même radical, les noms Dòbrńa, Dòbrńe, Dobrńca et v. serbo-cr. Dobrańa (ARj II 516). En tchèque, on trouve à côte de Dobrný, Dobrná, Dobrnik aussi Debrný, écrit Doberni en 1297 (Gebauer, SS I 215). Liewehr, Hist. Gramm. der tschech. Spr. 152 et 170, ainsi que ZfslPh XI 377, évoque, lorsqu'il parle de ces noms; le passage - $e$ - $>-o$ - dans le voisinage des labiales. En ce qui concerne les noms polonais Dobiernica à côté de Debrnica, Dobrzynka, 1491 Debrznyca, Rozwadowski (Studia 52) conclut à une étymologie populaire qui aurait rapproché ces noms du type Dobra, tandis qu’il admet 
pour les noms allemands Dobrin, Döbernitz la possibilité que leur -o- ait été substitué à l'ancien -z-, ce qui pourtant est peu vraisemblable, cf. Trautmann, Elb. I 32.

Le nom commun dial. slovène dobra scontrée où l'eau abondes, conservé à Pohorje, nous aide à expliquer d'une manière satisfaisante ce type de nom. Tandis que les noms Deber, Dabar etc. en slovène et en serbocroate n'apparaissent jamais dans les hydronymes - s.-cr. Dabrooac, Dabrovica, Dabroonica viennent de däbar scastors, et le ruisseau slovène Daberščak tire son nom d'un nom de propriétaire - le type Dobrona est un hydronyme fréquent; le toponyme slovène Dobrnič à Dobrniško polje dans la Suha Krajina, ainsi que la station thermale Dobrna sont caractérisés par une abondance de sources. Si l'on prend en considération aussi les noms sud-slaves, nous sommes obligés de conclure que la voyelle -odans ce type n'est pas le résultat d'une évolution relativement récente, mais qu'elle vient du slave primitif dobra qu'il faut distinguer du radical $d_{a} b r z$, dabro «gorge crevasse, vallée».

Il est pourtant difficile d'établir dans quelle mesure les nombreux noms slaves de rivières Dobra se ratiachent au nom dobra. L'ancien rapport - - - : $-o$ - trouverait une confirmation dans le nom de la rivière polonaise Brda, all. Brahe, noté pour la première fois en 1145 comme Dbra $<{ }^{*} d z b r a$, avec son affluent Zbrzyca, all. Spritze, Sbritze (Rozwadowski, Almae matri Jag., 1900, 110 et Studia 52) que l'on fait d'habitude dériver du préslave *dubra (Pokorny, IEW 264). Le fait cependant que, dans l'hydronymie slave, on ne trouve jamais le type de nom Dobro, Dobrzjo (= ruisseau), mais seulement le type Dobra, est digne de considération. Kozierowski, Badania IV 177 cite même le nom de lac Dobra, ef. pomér. 1281 stagnum Dobra, 1290 See Dobra (Trautmann ELB II 35) où l'on s'attendrait à un neutre s'il s'agissait d'un mot dérivé de dobrz sbons. L'alternance des types de nom Dobrica : Debrica mérite elle aussi notre attention, cf. le nom de lieu dit slovène Dobrica, s.-cr. Dobrica, pol. Dobrzyca, pomér. Döberitz, Doberitz (Trautmann, Elb. II 55) contre pol. Zbrzyca, s.-cr. Dabrica, grec moderne Aißeırar' (Vasmer, Sl. in Griechenl. 152), ainsi que le nom commun ukr. debrýcja, debryńećs storrent, ruisseau de forêts (Hrabec, Nazmy 57; Rozwadowski, Studia 54). Parmi les noms de rivière russes on trouve naturellement aussi le type Dobraja (Maštakov, Dnjepr 246), slovaque Dobrá (Šmilauer, Yodopis 463) et s.-cr. Dobra qui s'écrit en 1209 Dobra, en 1210 Dobro reka (Smičiklas, Cód. dipl. IIl 92 et 97). En partie, cela peut résulter d'une étymologie populaire. Le type de nom Dobra ooda et des noms qui lui ressemblent s'explique tout seul, 
lorsqu'il s'agit de petites sources à eau potable, comme p. ex. s.-cr. 1340 Dobrizdenecz (Smičiklas, Cod. dipl. X 419), et de villages établis dans leurs environs; les hydronymes du type pol. Dobromodna, russe Dobrovodje ont passé du village à la rivière. En territoire slovène, on trouve le type Dobra, cf. 1208 ad caput aque Dobra, 1213 rioulus Dobra (F. Kos, Gradioo V 900 et 901); en Haute-Autriche Tobra, 1142 Tabra, 1296 Dobra, 1370 Tabaraha (Schnetz, JA XXXIX 182), au Tyrol Täber et Taberer (Mitterrutzner, Progr. Gymn. Brixen 1879), à côté du ruisseau Dobršnik en Haute-Carniole, 1073 de rioo Tobropotoch, quod teutonice Guetback (dicitur) (F. Kos, Gradioo III 274), où cependant le nom actuel nous fait présumer que ce nom soit dérivé d'un anthroponyme.

Du point de vue de l'étymologie, l'appell. dobra appartient presque assurément au groupe de $d_{\bar{\imath}} b r \iota / d_{\imath} b r b$, lette dubra «mare», lith. dùburas «endroit creusé par l'eau < i.-e. *dh(e)ub- (Vasmer, REW I 333). En ce qui concerne le celt. primitif *dubro-, $-\bar{a},-i s$, irl. moyen dobur, gallois $d r f r$ ceau dans les noms Dover $<$ Dubris et Uernodubrum, les étymologistes hésitent entre *dh(e)ub- «profond s et *dheubh- snoir» (Pokorny, IEW 264; in extenso avec bibliographie, M. Förster, Themse $724 \mathrm{~s}$.), on pourrait cependant prendre en considération aussi le parallèle avec slave $d_{z} b r$ (Pokorny, $Z f c P h X X$ 63). Il y faut ajouter encore all. die Tauber (Springer, Die Flußnamen Würtenbergs und Badens, 1930, 62), la glosse

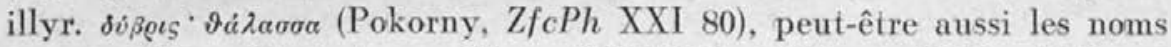
toscans Tubra (Kretschmer, Glotta XXII 216) et Tiberis (Oštir, Arhio za arb. star. I 83 et II 375), si l'on prend en considération la possibilité d'une explication préindo-européenne de ce nom.

Il est pourtant difficile de juger, si le rapport -o- :-z- :-b- représente en effet une innovation slave ou bien un héritage. Berneker explique la forme dubrı à côté de dzbrı par l'assimilation, il cite cependant la forme lette dubẹns à côté de dibẹns «fond, profondeurs. Quant à la forme en -o-, nous manquons de parallèles. J'attire l'attention sur le nom régional anglais Deira en Yorkshire qu'on décrit au $\mathrm{X}^{\mathrm{e}}$ siècle dans une traduction comme «terrain humides < v. bret. "Dobriā acontrée où l'eau abondes, ce qu'on interprète le plus souvent comme un dérivé du plur. v. bret. "doorä de *duvr «eaus (M. Förster, Themse 83 et 89). Là où l'inflexion celtique est relativèment récente, elle ne peut. guère dater d'une époque où les Celtes avaient la possibilité d'un contact avec les Slaves. Et pourtant la similitude sémantique et phonétique des bases celtique et slave est frappante. 
Les exemples tirés de la toponymie montrent que le nom commun dobra conservé à Pohorje est un reliquat slave; bien qu'il soit difficile de déterminer exactement ce nom et son étymologie, nous sommes d'avis qu'une révision des opinions actuelles s'impose.

\section{SLOVENE DIALECTAL PEN \&CIME >}

Le nom commun pen «cimes (Pohorski, Novice, 1859, 108) s'expliquerait difficilement en partant d'une base slave. Le terme v. sl. pъń «truncuss, slovène $p a ̂ n j$ esouche, ruches ne semble pas venir en considération, puisque les lois phonétiques en feraient à Pohorje * $p e ̂ j^{n}$; en Styrie orientale, ce terme a donné $p \stackrel{\varphi}{\eta} j^{n}, p e j^{n}{ }^{n} i c ̌$; toutefois, le passage sémantique «cime» $\longleftrightarrow$ «souche» est fort possible, cf. frioul. mùcola «collicello : basq. mokor, mukuŕ esouches ou sard. tuturru esouche, sommet» (J. Hubschmid, Sard. Stud. 50 et 78). On ne pourrait non plus songer à la base pẹti, pono $<*(s)$ pen-, puisque de tels dérivés ne se rencontrent point dans les langues slaves. L'idée d'un substrat semble s'imposer, mais nous ne devons pas conclure à la hâte au terme celtique penn stête, sommets (Holder II 966) puisque, jusqu'à ce jour, on n'a pas trouvé en Slovénie de reliquat celtique direct, et que ce nom celtique présente aussi des problèmes qui ne sont pas encore suffisamment éclaircis.

Du point de vue phonétique et de celui sémantique cependant, notre nom est fort proche du nom allemand de montagne Finne f. (chez Unstrut, Saale in Ilm) qui s'écrit en 1106 Vin silva, 1166 Vinna, 1382 Vyn (cité d'après A. Bach, Deutsche Namenkunde II 2, 44); plusieurs auteurs ont donné des explications diverses de ce nom. Si l'on laisse de côté les étymologies germaniques qui, du point de vue sémantique, présentent de grandes difficultés, comme p. ex. la comparaison avec Fischfinne ou avec v. h. all. fenna «marais» etc. (p. ex. O. Weise, GRM II 435, ou, autrement, F. Mentz, ZONF X 78), on trouve dans la littérature linguistique deux explications. De nombreux auteurs concluent au terme celtique penn (G. Kossina, PBB XX 296; A. Hirt, Etym. d. nhd. Spr. 382; F. Solmsen, Idg. Eigennamen 81) qui cependant aurait dû passer dans la langue germanique avant la mutation consonantique germanique R. Much chez Hoops, RL III 25, et K. B. Jensen, Actes et Mémoires I ${ }^{e r}$ Congrès, 1959, 155, sont cependant d'avis que all. Finne rèprésente le réflexe germanique régulier d'i.-e. "pinno- sbout, pointe, sommets, cf. lat. p̌nna (MeyerLübke, $R E W^{3}$ 6514/2). 
Toutes ces explications présentent des difficultés. Le terme v. irl. cenn, kymr. penn, exige une forme commune du celt. primit. "quen-, et non pas "ken- comme l'a présumé Walde-Pokorny I 398. On peut cependant douter que le mot celtique provienne en effet d'i.-e. *ken- ${ }^{1}{ }^{1}$; dans son nouveau $I E W$ 564, Pokorny ne cite plus cette étymologie problématique. On pourrait expliquer le celt. prim. "quen- aussi d'une forme i.-e. plus ancienne ou venant d'un substrat *pen- (Brugmann, Grundri $\beta$ I, $\$ 668)$. Vu l'étendue et l'obscurité de la famille de mots exclusivement romane punna, ef. v. it. penna «sommet», v. port. pena «roc», il serait également difficile de conclure à une forme i.-e. "pinno".

Les noms Apennini ne sont pas moins embarrassants. Outre dans le nom du massif italien, appelé de nos jours Apennino, en antiquité

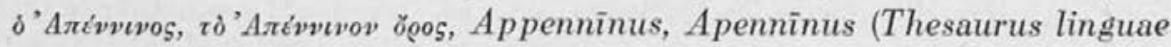
latinae II 278) qui n'a désigné primitivement que la partie nord-ouest, ligure, de ce massif (Huilsen dans PWRE II 1, 210 -214), cette base apparaît aussi dans le nom des Alpes Pennines (Poenina), en antiquité Alpe Pennino, Apennina, Alpes Poeninae, Alpes Appaenninae, etc. (Thesaurus,

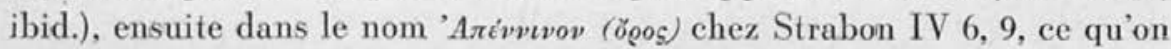
identifie le plus souvent avec le Brenner (Hopfer dans PWRE, Supl. III 129) et avec le toponyme actuel Pens. Dans le Derbyshire en Grande Bretagne aussi on trouve la Pennine Chain (Webster's Nero World Dictionary). Il n'est cependant pas éclairci si certaines citations anciennes du nom Apenninus ne se rapportent pas à des montagnes slovènes, ainsi que l'a présumé Pichler (Austria romana 111) d'après l'ordre des noms cités. Ce qui est certain, c'est que le passage suivant de Zosime coneerne

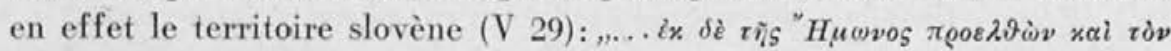

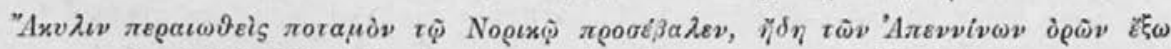
yevómevos..." On identifie les Apennins de ce passage d'habitude avec les montagnes dans les environs de Trojane ( J. Klemenc, Ptujski grad $v$ kasni antiki, 1950, 66).

Souvent, on explique ces noms aussi en partant de celt. penn stête, sommets (Terracini, AGI XX sez. Goid. 134-136) et d'un préfixe intensif ad-. De toutes les autres explications, je ne connais que celle d'Oštir (Etnolog II 58; Vogelnamen 61) qui suppose que cette base de toponyme soit paléoeuropéenne et qui y ramène, outre le terme slave (o)apıno «chaux», aussi etr. penəna «pierre, roc». Puisque le nom Apenninus dans l'inscription de 117 av. notre ère $C I L \mathrm{I}^{2}$ 584, V 7749 (voy. E. Diehl, Altlat. Inschriften 45) est cité parmi des noms exclusivement ligures, cette explication celtique reste pour le moins problématique. 
En territoire slovène, un nom qui mérite l'attention est le toponyme Opčine, it. Villa Opicina, Oppicina au-dessus de Trieste (slov. dial. ùopk'ine, loc. na uòpk'ịnz\%, nom d'habitant (u)opanci, adj. (u)oponski, Openski vrh, qui s'écrit en 1350 vallis de Opchiena, plus tard Opchena, Opechno (Kandler, CDI II). Si nous ne tenons pas compte des explications anciennes (selon Kandler < lat. Obsidianum, selon Trstenjak «vas na opaki strani hriba), voy. Sila, Trst in okolica 16), nous citerons seulement Skok qui pense que le nom roman est venu du slovène (Oko Trsta 176; Istoriski Casopis III, 1952, 30). Et pouriant le rapport surprenant entre la forme slovène du toponyme et entre le nom d'habitant et l'adjectif reste sans explication. Il faudrait reconstruire la forme slovène primitive *opsnt-: "opsn- avec le suffixe que l'on trouve dans le nom proche de Proačina près de Gorica. Cela nous mènerait au préslave (et peut-être même préroman) *ap(p)in(t)-/*ap(p)en(t)-. La situation de ce village sur la pente rocheuse au-dessus de la baie de Trieste parlerait en faveur de cette étymologie, bien que cette explication n'éclaircisse pas tous les problèmes.

On pourrait conclure qu'il y a entre le nom slov, pen scimes, all. Finne, prérom. $A p(p)$ ennīni et celt. penn $<{ }^{*} q u e n-$, et peut-être même lat. p̌nna un rapport qui indiquerait une parenté paléoeuropéenne.

\section{TCHEQUE DIALECTAL OTEN \&CLOTURE,}

Depuis Miklošič déjà, $E W$ 370, tous les dictionnaires étymologiques slaves (cf. A. Brückner, SEJP 589; Holub-Kopečný, ESJC 399) expliquent le sląve tynz cmur, clôtures comme un emprunt au germ. "tüna- (ou *tūnu), mais personne n'a encore étudié en détail cette intéressante famille de mots. A côté de v. sl. tynō emurus slovène tìn, tína sparoi de séparation, cloison, cloison de planches dans une écuries, s.-er. tin eclôtures, russe et ukr. tyn «clayonnages, pol. tyn <clôture, murs, tch. týn «enclos», slovaque týn «ais, planche», il faut considérer aussi les verbes: slovène zatiniti, -tinim «revêtir de planches», s.-cr. tiniti, obtiniti, zatiniti, tch. zatýniti, slovaque týnit, russe tynit, ukr. obtyniti, puis des dérivés comme p. ex. tch. týnka, pol. dial. tynianka, pomér. maten < *o-tynø, ainsi que les formes avec -b-, slov. tinj cplanches, tînjeoje ssuite de planches», russe tynı, tch. dial, zatyň, etc. Un terme qui mérite une attention particulière est le tchèque oteň, gen. otně cclôtures (Kott, Sl. II 442) qui nous fait supposer un $-\succsim-<-\breve{u}-$ ancien!

Un nom commun dialectal isolé pourrait naturellement résulter d'une évolution irrégulière. Un *otznı ancien est cependant attesté dans la to- 
ponymie de vastes territoires slaves. Outre des toponymes comme p. ex. tch. Tyniec, Tynice, Tyniště, pol. Tyniec, all. Thyn et, moins sûr, s.-cr. Tin (Mažuranić, Prinosi 1452), slovène Tinsko, 1404 Tinsk, 1436 Tinczka (Zahn, ONB 135), R. Trautmann (Die Elb- und Ostseeslav. ON II 68) nota aussi les noms Wotenitz (1250 Wotenist, 1507 Woteniste), Woeten (1328 Wotne), Wuthenom (1419 Wotenome, 1401 Ottenom), Wotenik (1186 Wteneke, 1309 Woteneke), et le lac Wutenitz. Pour expliquer ces noms, il faut partir de la base *o-tznb; les toponymes serbo-croates Otań, gen. Otŕa, loc. u Otńu, semblent venir de la même source: $A R j$ IX 348 cite deux villages de ce nom, Otań (aussi Hotań) en Hercegovina et Otań en Serbie près d'Užice. La forme Hotań ( $A R j$ II 655 ) doit résulter d'une orthographe pédantesque, puisque la déclinaison de ce nom ne permet pas de supposer un dérivé possessif d'un anthroponyme *Hotan. Il reste cependant douteux que l'on puisse dériver de la même base aussi les noms slovènes Otem et Otemna, dial. âutana (SR VIII 15), bien que la toponymie slovène présente des exemples de finale $-n-/-n-1\rangle-m-$, p. ex. Ćedem près de Brežice et Ščedem dans la vallée de Rož (Šašelj, Sooboda, 1951, 54) contre Ščedna, litt. Škedenj près de Trieste (Jelinčič, Primorski dneonik IX, 12 sept. 1953, 3-4) ou Śčädna près- de Solkan, et d'autres encore.

Il y a dans la toponymie slave beaucoup d'exemples où l'on trouve pour l'un ou l'autre type de nom propre un nom commun correspondant dans une région éloignée des territoires slaves; je me borne à citer le slovène $U d m a t$, s.-cr. Odmut : russe nom c. ómut, pol. odmęt emare» (SR V-VII 137); slovène Kujavič, s.-cr. Kujava, Kujavica, etc. : nom c. pol. kujarma «miejsce $\mathrm{v}$ polu jałowes, chez les Hutsules kújeroa «puste, odludne miejsces (Hrabec, Nazmy 82); slovène Knej, Kneja, s.-cr. Kneja : nom c. pol. knieja, russe kneja forêts (Dickenmann, AECO VII 177). De ce fait, la comparaison du s.-cr. Otań : tch. oteň est irréprochable du point de vue de la slavistique.

Les exemples de toponymie attestent assez clairement que le nom commun *o-tzńb est fort ancien et qu'il était très répandu. Il est donc impossible de l'expliquer comme un emprunt au germ. "tünu qui est attesté toujours avec un $-\bar{u}$ - long, cf. v. h. all. zūn cenceintes, v. nord. tūn «pré enclos, domaine, villages, ags. tūn cclôture, jardin, cour, villages, angl. tomn kmétairies, fris. tūn «jardin enclos près de la maisons, etc. Le russe synž cturris» < zūn (Vaillant, RÉSl XVI 99) cependant peut très bien représenter un emprunt germanique; le tch. týn echâteau, château forts, p. ex. Karlůs týn, peut avoir subi une influence germanique. 
La plupart des auteurs (v. M. Förster, Themse 36 avec bibliographie) sont d'avis que le mot germanique n'est pas un emprunt au celt. dūnum, cf. v. irl. dūn schâteau fort, ville fortifiée», dūnaim s'enferme, je fortifies, kymr. din schâteau forts, etc., mais concluent à une parenté primitive des deux bases celtique et germanique. L'analyse toponymique celtique et germanique confirme cette théorie (v. K. B. Jensen, Isoglosses toponymiques en celtique et en germanique. Classica et Mediaeoalia X, 1949, 165 d., et A. Bach, Deutsche Namenkunde II 2, 487).

Le slave tyń : tõn *d/tŭn(n)-. Ce phénomène serait difficile à expliquer par la phonétique indo-européenne. L'étymologie indo-européenne de ce groupe de mots était d'ailleurs toujours douteuse (Walde-Pokorny I 778), et Pokorny a abandonné dans son IEW. sa théorie ancienne. Oštir (Arhio za arb. star. I 115) propose un pré-i.-e. * $\Theta o ́ n$ et le confronte avec les éléments

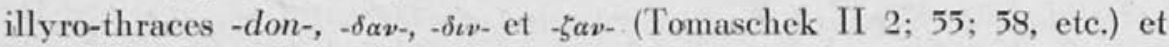
mi-dne cvicus». Ce qui est sûr, c'est que le slave o-tonı s'oppose aux explications de ce mot de civilisation très ancien qu'on a données jusqu’à présent.

\section{Porzetek}

\section{Leksični doneski}

1. Sloven. dial. dobra seine wasserreiche Gegends bi bilo mogoče vezati z dъbrь, dьbrь zaradi imen sloven. Dobrna, Dobrnica, Dobrnič poleg Debrnica, češ. Dobrná, Dobrný, Dobrnik poleg Debrní, polj. Dobiernica poleg Debrnica itd. Morda spadajo sem tudi rečna imena Dobra poleg polj. Brda $<{ }^{*} D_{\text {Ł }} b r a$. Razmerje -o- :- $\mathrm{b}-:-\mathrm{b}-\mathrm{V}$ tej osnovi je komaj slovansko, prim. letsko dubẹns : dibẹns; britansko keltsko pokrajinsko ime Deira $<{ }^{*}$ Dooria , Wasserlande je po vokalizmu in semantično presenetljivo blizu tej slovanski imenski bazi, ki se je na Pohorju ohranila kot apelativ.

2. Sloven. dial. pen sBergspitze spominja fonetiěno in semantiěno na nemška gorska imena Finne $\mathrm{f}$. in na imena $A p(p)$ ennini, kar oboje razlagajo iz keltskega penn >Kopf, Gipfele. Ta keltska beseda more biti samo iz starejšega prakeltskega "quen-. Ni pa izključeno, da je prakeltsko *quen- iz še starejšega "pen-, ki pa zaradi romanskega pinna sipfele ne bi mogel biti rezultat regularnega idevr. razvoja, ampak bi kazal na substrat. Slovensko ime Opčine, adj.

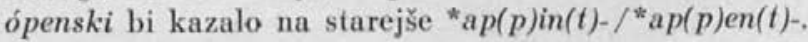

3. Ceš. dial. oteň sZaun s je zaradi imen pomorj. Wotenitz, Woeten, Wotenik, Wutenitz in shrv. Otań, morda tudi sloven. Otem, Otemna (ako je izglasni $m<n ́$ )

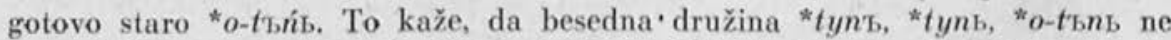
more biti prevzeta iz germ. "tünu, kot so mislili doslej, ampak kaže samo na sorodnost s keltskim dünum in germ. " tūnu ter govori za praobliko *d/tūn- : *d/tŭn(n)-, kar se ne bi moglo zadovoljivo razložiti z idevr. glasoslovjem. 